\title{
Design of Tunable Equalizers Using Multilayered Half Mode Substrate Integrated Waveguide Structures Added Absorbing Pillars
}

\author{
Shuxing Wang, ${ }^{1}$ Yongfei Wang, ${ }^{1}$ Dewei Zhang, ${ }^{1}$ Yi Zhang, ${ }^{1,2}$ and Dongfang Zhou ${ }^{1}$ \\ ${ }^{1}$ Department of Electromagnetic Wave and Antenna Propagation, Institute of Information Science and Technology of Zhengzhou, \\ Zhengzhou, Henan 450001, China \\ ${ }^{2}$ Microwave Tech and Antenna, Department of Electronic Engineering, Tsinghua University, Beijing 100084, China
}

Correspondence should be addressed to Shuxing Wang; wangshuxing000@126.com

Received 7 June 2015; Revised 17 September 2015; Accepted 28 September 2015

Academic Editor: Giovanni Berselli

Copyright (C) 2015 Shuxing Wang et al. This is an open access article distributed under the Creative Commons Attribution License, which permits unrestricted use, distribution, and reproduction in any medium, provided the original work is properly cited.

\begin{abstract}
An equalizer based on multilayered half mode substrate integrated waveguide (HMSIW) structures with high Q-factor, low loss, and compact size is proposed for the first time. Resonant cavities distributing in the upper substrate and the bottom substrate, with the middle substrate layer which works as the transmission line together, constitute a multilayer structure. The design method and theoretical analysis are summarized first. The mode analysis, simulated results, and measured results are all provided. The measured results show a good performance and are in agreement with the simulated results, and the maximum attenuation slope reaches $-16 \mathrm{~dB}$ over $12.5 \mathrm{GHz} 14.5 \mathrm{GHz}$. With the use of absorbing pillars, the attenuation and $Q$ value can be tuned more easily than the other planar equalizers. Compared with the SIW equalizer, the size of this structure reduces by $50 \%$. Furthermore, this structure is suitable for the miniaturization development of equalizers.
\end{abstract}

\section{Introduction}

The equalizers are used to compensate the output gain slope fluctuation of traveling wave tube amplifiers (TWTAs) in the radar systems [1]. The equalizers are placed mostly between the preamplifier and the postequipped TWTA rather than the back-end of TWTA due to the high output power of TWTA. The preamplifier provides the same exciting power at each frequency point for the post-TWTA. In this way, the output gain of TWTA has a large fluctuation, whereas the output power of TWTA we need is the same at each frequency point. The equalizer, whose attenuation frequency characteristics are complementary with the output power characteristics of TWTA in form, is designed to transform the original exciting power into the optimal exciting power required by TWTA to get the best output power. Equalizers play an important role in improving the detection distance of radars. $Q$ value is the ratio of the energy stored in the resonant cavity to the energy loss of each cycle and it is an important parameter in resonant circuits. The bigger the $Q$ value is, the more energy the resonant cavity stores. The equalizers with high $Q$ value can be used in high power amplifier system.

Nowadays, equalizers in rectangular waveguide type loaded coaxial cavity (narrow bandwidth) and planar type loaded open resonant stub (relatively wide bandwidth) have been applied in lots of microwave and millimeter systems [26]. On the other hand, the integrated level of RF, microwave, and millimeter wave circuits is getting higher and the size of components is getting smaller due to the rapid development of new materials and new technology. The miniaturization has become the development trend of passive microwave components, including equalizers [7]. The demand for equalizers with small size, low loss, and high $Q$ value grows gradually. The traditional equalizers, such as coaxial cavity equalizers with large size and microstrip equalizers with high insertion loss and low $Q$ value, cannot meet the need of TWTAs.

Substrate integrated waveguide (SIW) is a new planar electromagnetic guide wave structure and is better than the traditional microstrip and waveguide type for the superiority 
of the small size, high $Q$ value, and low insertion loss. Nowadays, this structure is being widely used in the design of microwave and millimeter wave devices [8-14]. In [12] a single layer SIW equalizer with large size is proposed whose attenuation cannot be tuned. In [13] an equalizer is fabricated using complex LTCC technology, and it has a large insertion loss because of the substrate's uneven surface. In [14] a dual layer equalizer is designed which cannot tune the attenuation and $Q$ value, and the probe excitation plays a poor role in coupling energy into cavities. What is worse, the size of SIW structure is still difficult to meet the requirement of the equalizer's miniaturization.

Compared with SIW, Half mode substrate integrated waveguide (HMSIW) has a smaller size but with the same performance, and the integrated degree of the substrate integrated waveguide type device is improved [15]. This structure has been widely used in microwave and millimeter wave devices [16, 17] except equalizers.

Based on [14] written by authors, for the first time, an equalizer based on multilayered HMSIW structures is designed and fabricated. It has six cascaded HMSIW resonators. Each of them serves as an independent attenuation tune substructure unit.

\section{Design Procedure and Analysis}

A linear array of metallized via holes in substrate, with the upper metallic surface and bottom metallic surface, constitutes the HMSIW structure. A substrate with dielectric constant 11.9 is selected in this paper to simulate and fabricate the equalizer. The thickness of substrate is $0.6 \mathrm{~mm}$.

2.1. The Design of HMSIW Transmission Line. To design the HMSIW equalizer, the HMSIW transmission line should be discussed first. It locates in the middle layer. This structure is used to transmit energy and to excite HMSIW cavities distributing in the upper and bottom layer. To only transit the dominant mode, $\mathrm{TE}_{10}$ mode, and to inhibit the highorder modes, the dimension of HMSIW should be calculated precisely.

HMSIW can be equivalent with the conventional rectangle waveguide; transformation equations are given as follows [18]:

$$
\begin{aligned}
& W_{e}=\frac{1}{2}\left(W-1.08 \frac{D^{2}}{b}+0.1 \frac{D^{2}}{W}\right), \\
& L_{e}=L-1.08 \frac{D^{2}}{b}+0.1 \frac{D^{2}}{W},
\end{aligned}
$$

where $W$ and $L$ are the length and width of HMSIW, respectively, and $W_{e}$ and $L_{e}$ are the width and length of rectangle waveguide, respectively. $D$ is the diameter of via hole and $b$ is the distance between via holes. When $b / D<2$ and $D / W<0.2$, the rectangle waveguide resonant cavity theory can be applied to the design of HMSIW resonant cavity. As the HMSIW transmission line is excited by a microstrip line, the microstrip-to-HMSIW transition is designed to guarantee the impendence matching. It is the characteristic impedance rather than the wave impedance that should be focused. The equivalent characteristic impedance of HMSIW derived from the characteristic impedance of conventional rectangular waveguide [19] is given in (4).

The equation of characteristic impedance of conventional rectangular waveguide is as follows:

$$
Z_{e}=\frac{\pi b}{2 a} \sqrt{\frac{\mu}{\varepsilon}} \frac{1}{\sqrt{1-(\lambda / 2 a)^{2}}}
$$

where $a$ and $b$ are the width and height of conventional rectangular waveguide, respectively, $\mu$ is magnetic permeability, $\varepsilon$ is dielectric constant, and $\lambda$ is working wavelength.

We replace $a$ and $b$ with the height $h$ and the equivalent width $W_{e}$ of HMSIW, respectively. Using the equation,

$$
\sqrt{\frac{\mu}{\varepsilon}}=\frac{\eta}{\sqrt{\varepsilon_{r}}}
$$

where $\eta$ is the wave impedance, we get

$$
Z_{e}=\frac{\pi \eta_{o} h}{4 W_{e} \sqrt{\varepsilon_{r}\left[1-\left(\lambda / 4 W_{e}\right)^{2}\right]}},
$$

where $\eta_{o}=120 \pi(\Omega)$ is the wave impedance of TEM mode in the air, $h$ is the height of the dielectric substrate, and $\varepsilon_{r}$ is relative permittivity.

The microstrip line is well suited to excite the waveguide because the electric fields of the two dissimilar structures are approximately oriented in the same direction and also they share the same profile.

Multiple microstrip lines with different widths and lengths are adopted in this paper to design the transition. The main idea is (1) getting the impedance of HMSIW by (4) and then making it equal to the impedance of microstrip; (2) using the microstrip transmission line impedance equation to calculate the width of a single tapered transmission line; and (3) giving the length of this line segment and calculating the input impedance and repeating the steps above, until the input impedance nearly reaches $50 \Omega$.

Figure 1 shows the measured and the simulated result of the proposed transition made up of five microstrip segments. The maximum measured insertion loss is nearly $0.15 \mathrm{~dB}$ in the entire band, better than $0.7 \mathrm{~dB}$ in [19].

2.3. The Analysis of HMSIW Resonant Cavity. The definition of TE mode or TM mode does not depend on the coordinate axis, whereas the definition of the $\mathrm{TE}_{m n}$ and $\mathrm{TM}_{m n}$ mode is relative, related to the coordinate axis. The equivalent structure of HMSIW cavity unit is illustrated in Figure 2.

Based on the black coordinate axis, the dominant mode is $\mathrm{TE}_{101}$ mode, and it has three components: $E_{y}, H_{x}$, and $H_{z}$. In accordance with this red coordinate axis which is obtained by the rotation of black coordinate axis, the three components of 


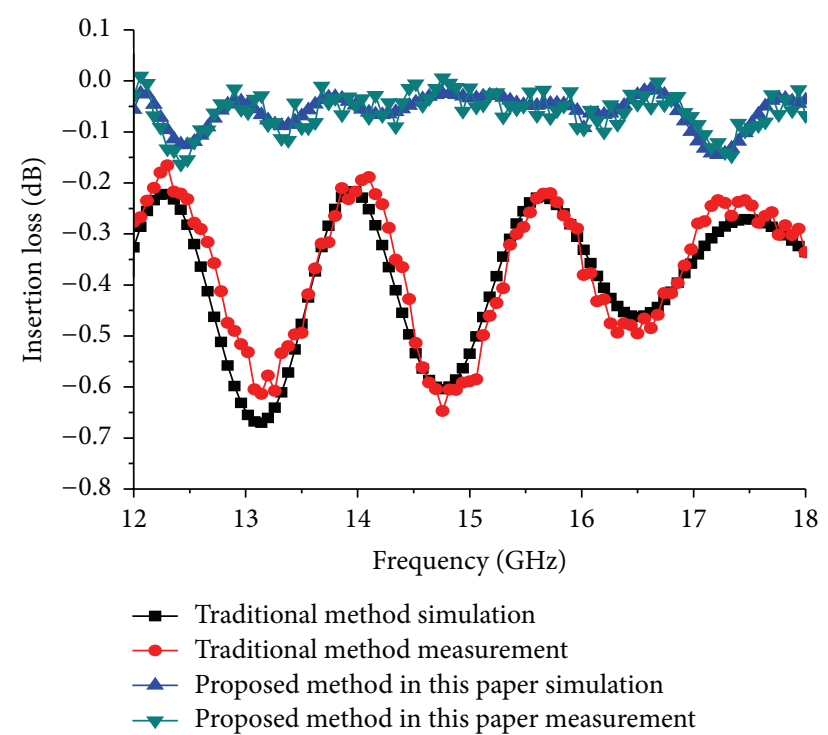

FIGURE 1: Simulated and measured insertion loss $S_{21}$ of the transition.

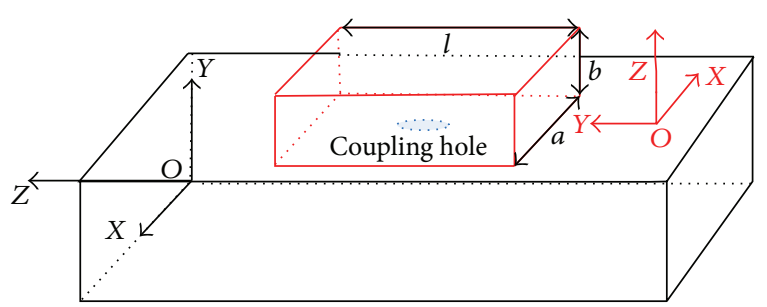

FIgURE 2: The equivalent resonant cavity of HMSIW.

$\mathrm{TE}_{101}$ mode have changed as follows; namely, the $\mathrm{TE}_{101}$ mode becomes the $\mathrm{TM}_{110}$ mode:

\begin{tabular}{|ccc|}
\hline $\mathrm{TE}$ & & $\mathrm{TM}$ \\
$\left(\mathrm{TE}_{101}\right)$ & & $\left(\mathrm{TM}_{110}\right)$ \\
$E_{y}$ & $\longrightarrow$ & $E_{z}$ \\
$H_{x}$ & $\longrightarrow$ & $H_{x}$ \\
$H_{z}$ & $\longrightarrow$ & $H_{y}$ \\
\hline
\end{tabular}

In fact, the field distributions of the two modes are identical. But it is the $\mathrm{TM}_{110}$ mode rather than the $\mathrm{TE}_{101}$ mode that should be chosen to calculate the resonant frequency; otherwise, there will be a mistake.

The resonant frequency is calculated by (6), and the height of HMSIW resonant cavity can reach $0 \mathrm{~mm}$ [20]:

$$
f\left(\mathrm{TM}_{110}\right)=\frac{c_{0}}{2 \sqrt{\varepsilon_{r}}} \sqrt{\left(\frac{1}{L_{e}}\right)^{2}+\left(\frac{1}{2 W_{e}}\right)^{2}},
$$

where $c_{0}$ is the light velocity in vacuum. The high-order modes should be restrained to make these cavities work in the $\mathrm{TM}_{110}$ mode. This paper proposes a method to restrain the high-order modes:

$$
\begin{aligned}
& \frac{c \pi}{\sqrt{\varepsilon_{r} \mu_{r}}} \sqrt{\left(\frac{1}{2 W_{e}}\right)^{2}+\left(\frac{2}{L_{e}}\right)^{2}} \geq \omega_{2}, \\
& \omega_{1} \leq \frac{c \pi}{\sqrt{\varepsilon_{r} \mu_{r}}} \sqrt{\left(\frac{1}{2 W_{e}}\right)^{2}+\left(\frac{1}{L_{e}}\right)^{2}} \leq \omega_{2}, \\
& \frac{c \pi}{\sqrt{\varepsilon_{r} \mu_{r}}} \sqrt{\left(\frac{2}{2 W_{e}}\right)^{2}+\left(\frac{1}{L_{e}}\right)^{2}} \geq \omega_{2} .
\end{aligned}
$$

Suppose that the HMSIW resonant cavity works at $\left(\omega_{1}, \omega_{2}\right) ; \mu_{r}$ is relative permeability. Equations (7) and (9) mean the resonant frequencies of high-order modes are not in this band while (8) shows that the $\mathrm{TM}_{110}$ mode works in it. In this way, it makes sure that the cavity works in the $\mathrm{TM}_{110}$ mode.

The $\mathrm{TE}_{10}$ mode is transmitted by the HMSIW line and the energy enters into cavity through the coupling circle. Usually, the coupling is caused by $H_{x}, H_{z}$, and $E_{y}$ or a combination of them. The coupling in this structure is resulted from the $E_{y}$ component because the surface current is mainly in the $Y$ direction. The coupling circle is equivalent as a capacitive electrical susceptance $b_{y}$ in parallel [21]:

$$
b_{y}=\frac{B_{y}}{Y_{0}}=\frac{4 \pi \lambda_{g} P_{e y}}{a b \lambda_{0}^{2}} \sin ^{2}\left[\frac{\pi x}{a}\right],
$$

where $\lambda_{g}$ is the waveguide wavelength and $\lambda_{0}$ is the free space wavelength and $Y_{0}$ is the waveguide admittance of dominant mode. $P_{e y}$ is an electric polarization function of frequency and coupling circle's size. The electric-field component $E_{z}$ of $\mathrm{TM}_{110}$ is

$$
E_{z}=E_{0} \sin \left(\frac{\pi}{a} x\right) \sin \left(\frac{\pi}{l} y\right) .
$$

The $H_{x}$ and $H_{y}$ can be calculated by the Maxwell equation set as follows:

$$
\begin{aligned}
\nabla \times \mathbf{E} & =-j \omega_{0} \mu \mathbf{H}, \\
\mathbf{H} & =j \frac{1}{\omega_{0} \mu}\left|\begin{array}{ccc}
\vec{x} & \vec{y} & \vec{z} \\
\frac{\partial}{\partial x} & \frac{\partial}{\partial y} & \frac{\partial}{\partial z} \\
0 & 0 & E_{z}
\end{array}\right|,
\end{aligned}
$$

where $\omega_{0}=2 \pi f$ and $f$ is resonant frequency. The $Q$ value of HMSIW cavity should be concerned. The loss of HMSIW cavity is mainly due to the six metallic faces and the filled dielectric. Assuming that the loss of metallic faces is $P_{m}$, the dielectric loss is $P_{d}$, and the electric energy is $W_{e y}$, then the $Q$ is obtained:

$$
\begin{aligned}
Q & =\frac{2 \omega_{0} W_{e y}}{P_{m}+P_{d}}=\frac{1}{\left(\left(2 \pi^{2} /(k a l)^{3} b \eta\right) \sqrt{\mu \omega / 2 \sigma} u+\operatorname{tg} \sigma\right)}, \\
u & =\left(2 a^{3} b+2 b l^{3}+a^{3} l+a l^{3}\right),
\end{aligned}
$$




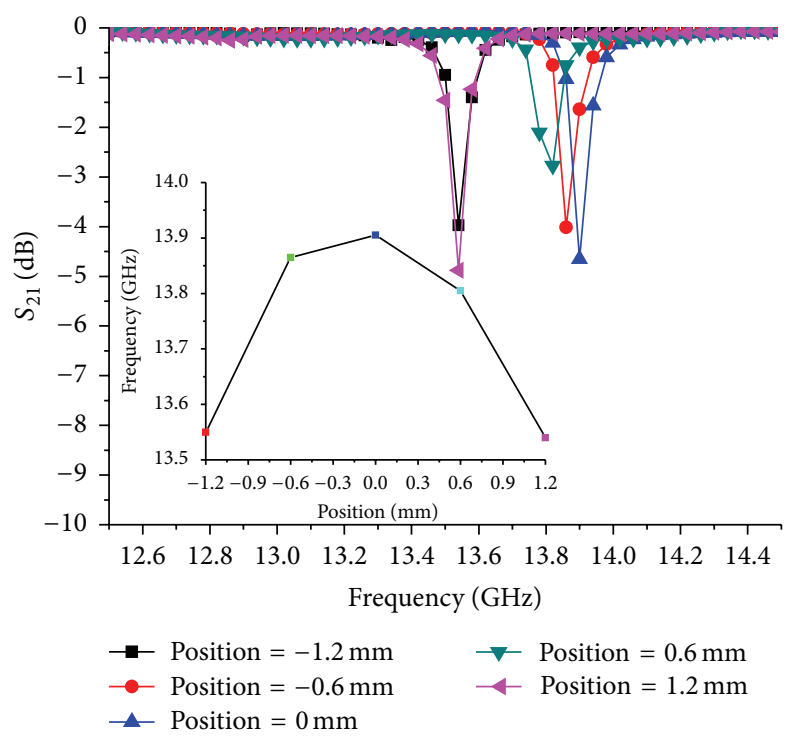

(a)

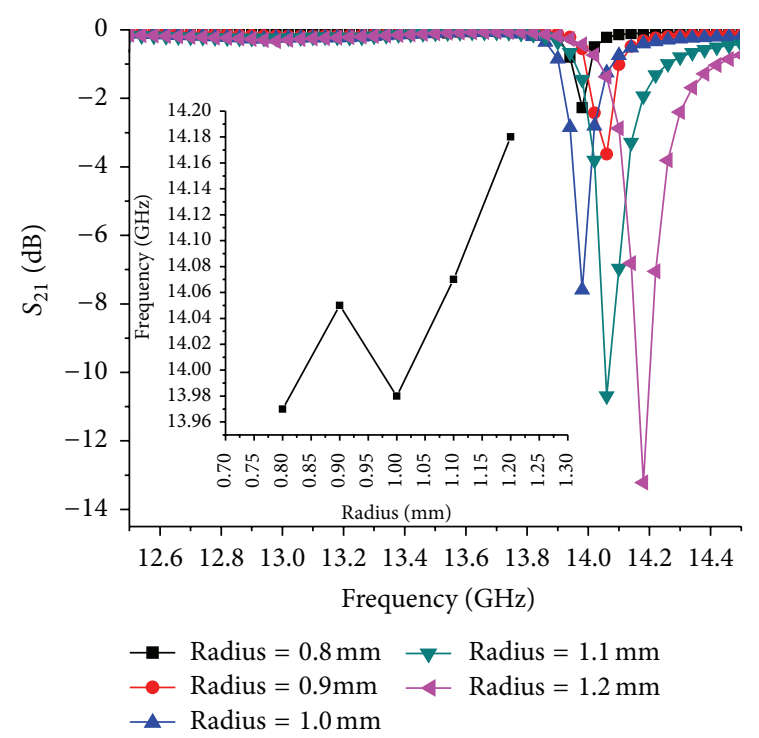

(b)

FIGURE 3: Simulation results of the HMSIW resonator. (a) Changes of frequency with different positions (in $y$-axis). (b) Changes of frequency with different radiuses.

where $k=\omega \sqrt{\mu \varepsilon}$ and $\operatorname{tg} \sigma$ is loss tangent. The $Q$ is determined by dielectric loss since the size of cavity is set already. On one hand, the larger the relative dielectric constant is, the larger the dielectric loss is. On the other hand, the dielectric constant determines the power capacity, and they are positively related. A proper dielectric should be chosen to keep a balance between the dielectric loss and power capacity.

2.4. The Design of Coupling Structures and Absorbing Pillars. To design the coupling circles, the position and radius are focused. Once the size of cavity is set, the cavity works at the dominant mode, $\mathrm{TM}_{110}$. We can excite the cavity at the middle of wide metal wall (along $y$-axis) or the narrow metal wall (along $x$-axis) to get the maximum field value, whereas the maximum value of $S_{21}$ should be limited. This paper solves this problem in two ways: one is to change the radius of coupling circle; another is to divert the optimal excitation position. The results are shown in Figure 3.

From Figure 3, we can know that the resonant frequency deviates when the position changes along $y$-axis. This is because the discontinuity at coupling circle excites the highorder modes and each mode has a unique attenuation distance, so different positions have different numbers of modes, which have a contribution to the generalized $S$ parameter matrix. The generalized $S$ parameter matrix is a matrix of infinite dimension, and it shows the mutual coupling between all modes. Usually, only the mutual coupling between the dominant modes is concerned, then the generalized $S$ parameter matrix is reduced to a two-dimensional matrix; namely,

$$
\mathbf{S}=\left[\begin{array}{ll}
S_{11} & S_{12} \\
S_{21} & S_{22}
\end{array}\right] .
$$

The change in radius of coupling circle can also cause the deviation of frequency because the coupling capacitance has

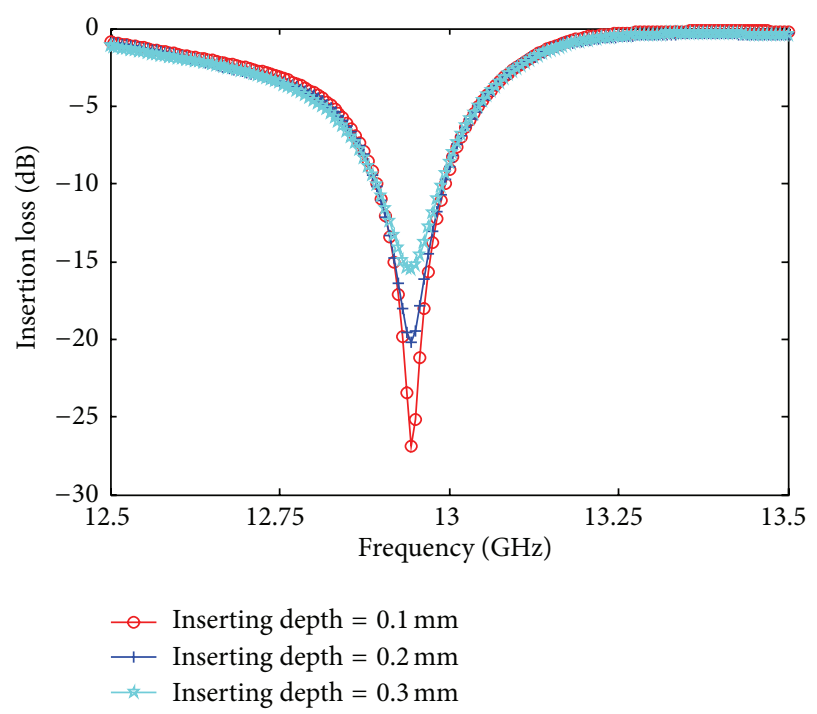

FIGURE 4: Changes of attenuation with different depths of absorbing pillar.

changed as the different sizes of holes are equivalent to the different coupling capacitances, shown in (10).

Most importantly, absorbing pillar arrays are used to tune the attenuation. The quantity, radius, position, and insertion depth of absorbing pillars are focused.

Figure 4 shows the result when the insertion depth changes. The other simulation results are omitted here for brevity. The absorbing pillars are filled by the hydroxyl iron, whose relative permittivity is 30 , dielectric loss tangent is 0.53 , relative permeability is 5 , and magnetic loss tangent is 0.38 .

Lots of air pillars can be dug in the HMSIW resonant cavities, and the air pillars can be filled with materials 
alternative, such as absorbing materials or dielectric substrate materials; it depends on the actual demand.

Film resistors can also be used to tune the attenuation. And the dimension of film resistors should be large enough, so film resistors can radiate heat efficiently. The equalization ability of film resistors is limited, no more than $15 \mathrm{~dB}$.

Through lots of simulations and calculations, we find that when the width of HMSIW resonator is fixed, the longer the length of resonant cavity is, the lower the resonant frequency is. In a word, the resonator length and the absorbing pillar parameters can affect frequency and attenuation regularly and respectively. The cascaded HMSIW cavities can meet the need of TWTAs.

2.5. The Design and Measurement of HMSIW Equalizer. With the analysis above, a HMSIW equalizer with six HMSIW cavities added absorbing pillars is simulated and fabricated. As the relative permittivity of substrate is 11.9, an equivalent magnetic wall is formed and it prevents the energy leaking from the long side wall without the metallic via holes.

Figure 5 shows the configuration of HMISW equalizer. Figure 5(a) illustrates the upper layers: it has three HMSIW resonant cavities and several absorbing pillars. Figure 5(b) shows the middle layer, it consists of two microstrip lines, two microstrip-to-HMSIW transitions, and a HMSIW line as well as three coupling circles. Figure 5(c) illustrates the ground layer, and the three HMSIW resonant cavities in the bottom layer are excited by the circles etched on the ground. Figure 5(d) shows the bottom layer and it has three resonant cavities. A coordinate axis has been given in Figure 5 for the convenience of illustrating the parameter position. $\left(x_{i}, y_{i}, r_{i}\right)$ represents the position and the radius of coupling circle and $L_{R i}$ represents the length of useful part of coupling circle. Figure 5(e) shows the side view of this equalizer while Figure 5(f) gives the whole structure. Figure 5(g) gives the photo of the fabricated sample.

All the values of parameters labeled in Figure 5 are shown in Table 1. This equalizer is fabricated with three PCB substrates. An Agilent N5244A network analyzer is used to verify its performance. Also, the traditional waveguide equalizer loaded by coaxial resonant cavities and the SIW equalizer loaded by six SIW resonant cavities are tested to compare with this structure. The insertion loss $S_{21}$ and the return loss $S_{11}$ are given in Figure 6. From Figure 6, we can know that the performance of the proposed HMSIW equalizer is nearly the same as that of the SIW equalizer, and it proves the conclusion in [15] perfectly. The maximum insertion loss of HMSIW equalizer is $1.4 \mathrm{~dB}$ while the maximum insertion loss of SIW equalizer is $1.1 \mathrm{~dB}$, and the maximum return loss of HMSIW is nearly $12.5 \mathrm{~dB}$ while that of the SIW is $12.3 \mathrm{~dB}$. The result shows that the HMSIW equalizer has a larger insertion loss. This is because one side wall of HMSIW equalizer has a slim energy leakage which is inevitable. The insertion loss of waveguide equalizer is nearly $2 \mathrm{~dB}$ with the maximum return loss of $18 \mathrm{~dB}$. With the use of isolator, the return loss of waveguide equalizer is better than others. The waveguide equalizer has an excellent tune quality compared to any other types, but waveguide equalizer has a large volume and is difficult to integrate with another planar equipment.
TABLE 1: The parameter values of the proposed equalizer.

\begin{tabular}{|c|c|}
\hline Parameter & Value $(\mathrm{mm})$ \\
\hline$W_{1}$ & 0.53 \\
\hline$L_{1}$ & 12.5 \\
\hline$W_{L}$ & 1.40 \\
\hline$W_{d 1}$ & 4.50 \\
\hline$W_{d 2}$ & 5.10 \\
\hline$W_{d 3}$ & 4.99 \\
\hline$W_{d 4}$ & 5.05 \\
\hline$W_{d 5}$ & 5.23 \\
\hline$W_{d 6}$ & 4.83 \\
\hline$b$ & 1.50 \\
\hline$D$ & 1.00 \\
\hline$R_{b i}$ & 0.30 \\
\hline$r_{1}$ & 0.90 \\
\hline$r_{2}$ & 1.10 \\
\hline$r_{3}$ & 0.95 \\
\hline$r_{4}$ & 0.95 \\
\hline$r_{5}$ & 1.00 \\
\hline$r_{6}$ & 0.90 \\
\hline$h$ & 0.60 \\
\hline$x_{1}$ & 2.50 \\
\hline$y_{1}$ & 26.00 \\
\hline$x_{2}$ & 2.50 \\
\hline$y_{2}$ & 30.80 \\
\hline$x_{3}$ & 2.10 \\
\hline$y_{3}$ & 35.70 \\
\hline$x_{4}$ & 2.50 \\
\hline$y_{4}$ & 37.50 \\
\hline$x_{5}$ & 2.50 \\
\hline$y_{5}$ & 30.30 \\
\hline$x_{6}$ & 2.50 \\
\hline$y_{6}$ & 37.50 \\
\hline$L_{R 1}$ & 0.90 \\
\hline$L_{R 2}$ & 1.10 \\
\hline$L_{R 3}$ & 1.35 \\
\hline$L_{R 4}$ & 0.95 \\
\hline$L_{R 5}$ & 1.00 \\
\hline$L_{R 6}$ & 1.20 \\
\hline$h_{R}$ & 0.20 \\
\hline
\end{tabular}

\section{Conclusion}

A novel equalizer based on multilayered HMSIW structures is proposed in this paper, working at $12.5 \sim 14.5 \mathrm{GHz}$, with small size, high $Q$ value, and low insertion loss. The measured result is in good agreement with the simulated result. It shares the same performance with the SIW equalizer only in half size, suitable for the development tendency of equalizers' miniaturization. This structure can also be used as the microwave band stop filter without absorbing pillars. 


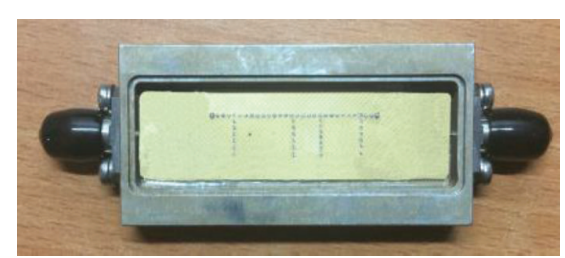

(g) Fabricated sample
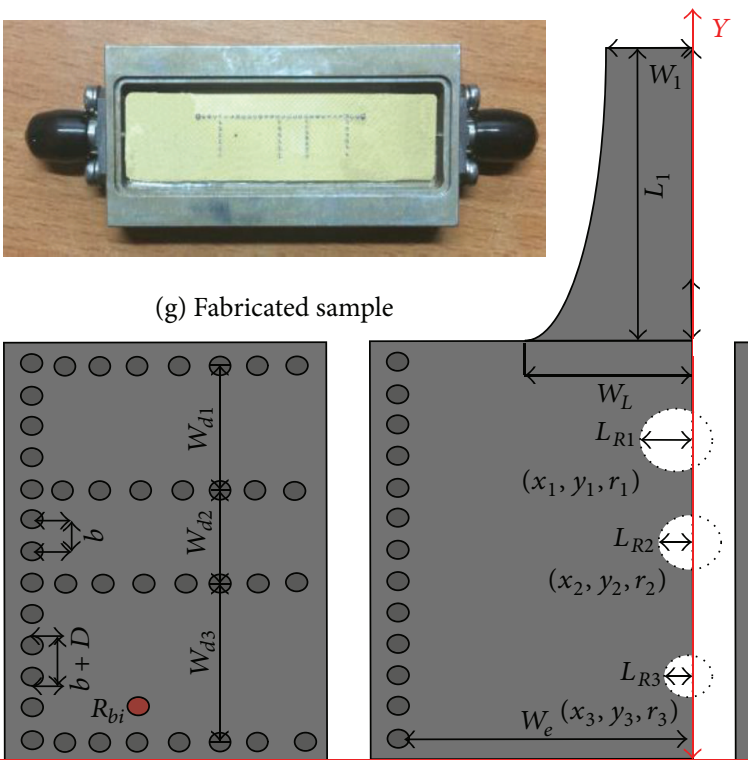

(a) Upper layer

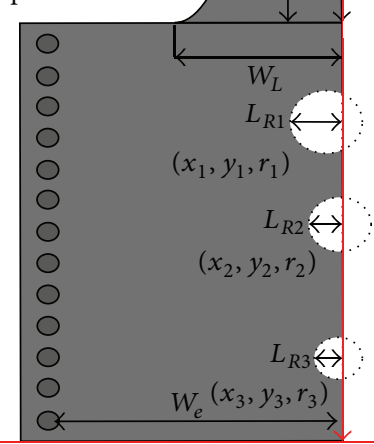

(b) Middle layer

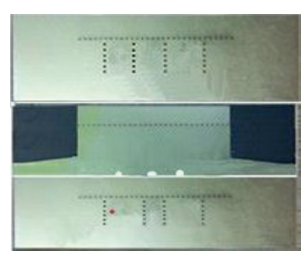

Photograph of three layers

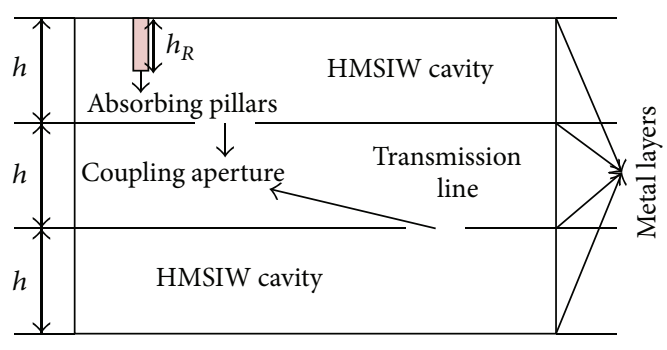

(e) Side view

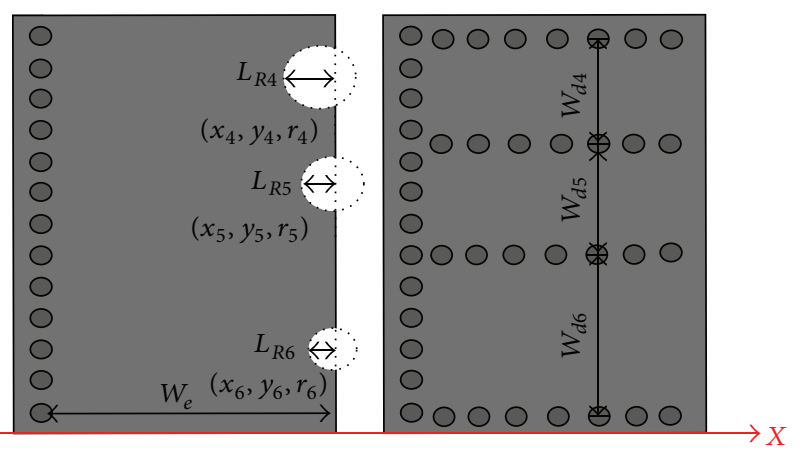

(c) Ground layer

(d) Bottom layer

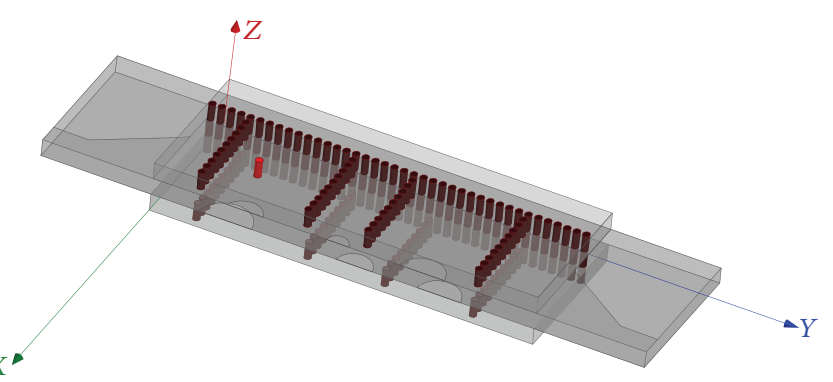

(f) The whole structure

Figure 5: Configuration of the proposed equalizer.

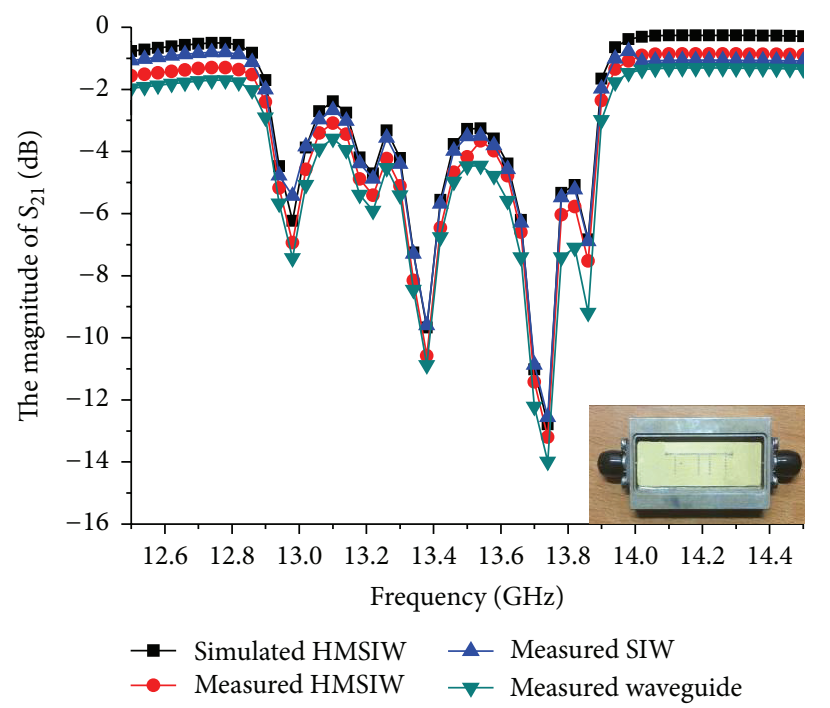

(a) The measured $S_{21}$ curves of different equalizers

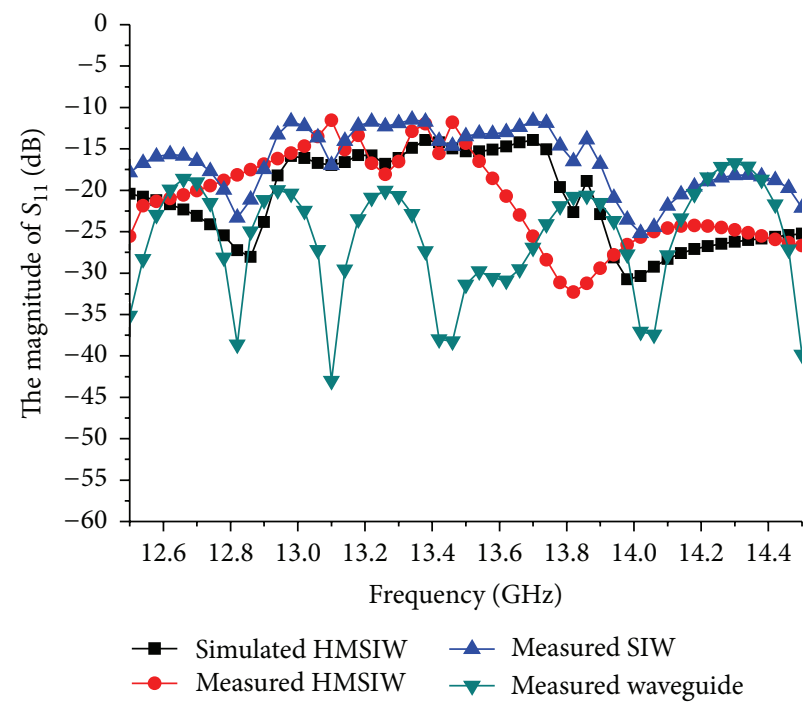

(b) The measured $S_{11}$ curves of different equalizers

FIGURE 6: Method proposed in this paper: simulation and measurement results. 


\section{Conflict of Interests}

The authors declare that there is no conflict of interests regarding the publication of this paper.

\section{Acknowledgments}

The authors would like to thank Dr. Zhang and Dr. Lv for their assistance in the theoretical guidance. This work is supported in part by the National Natural Science Foundation of China under Grant 62101056 and the Chinese Ministry of Industry and Information Technology and Chinese Ministry of Science under Grant 2015-ZX01010101-003

\section{References}

[1] H. Wei, "New progress and development trend of microwave theory and technology," Journal of Microwave, vol. 12, no. 4, pp. 341-344, 1996.

[2] D. F. Zhou, G. X. Sun, Z. X. Niu, J. P. Ren, and C. Lv, “The analyzing and optimization designing of high-power microwave equalizer," Vacuum Electronics, vol. 2, pp. 1-10, 2000.

[3] Y. Zhang, Z. Niu, and D. Zhou, "Design and implementation of a new type of millimeter wave microstrip equalizer," Journal of Infrared and Millimeter Waves, vol. 5, pp. 393-396, 2006.

[4] D. Zhang, D. Zhou, Z. Niu, and Z. Zhu, "Novel design of coaxial resonator microwave amplifier equalizer," Journal of Vacuum Science and Technology, vol. 28, no. 6, pp. 507-510, 2008.

[5] S. C. Bera, "Amplitude tilt active equalizer for frequency and temperature compensation," IEEE Microwave and Wireless Components Letters, vol. 21, no. 7, pp. 344-346, 2011.

[6] H. Wang, D. Zhou, Z. Niu, Z. Ren, and Z. Sun, "An objectoriented analysis of the high-power microwave equalizer simulation based on the measurement database," Vacuum Electronics, no. 1, pp. 24-26, 2003.

[7] W. Xuan, "Simulation and design of miniaturized millimeter wave gain equalizer," Telecommunication Engineering, vol. 47, no. 5, pp. 112-115, 2007.

[8] Y. S. Zhang and W. Hong, "A millimeter-wave gain enhanced multi-beam antenna based on a coplanar cylindrical dielectric lens," IEEE Transactions on Antennas and Propagation, vol. 60, no. 7, pp. 3485-3488, 2012.

[9] K. Gong, W. Hong, Y. Zhang, P. Chen, and C. J. You, "Substrate integrated waveguide quasi-elliptic filters with controllable electric and magnetic mixed coupling," IEEE Transactions on Microwave Theory and Techniques, vol. 60, no. 10, pp. 3071-3078, 2012.

[10] D.-F. Guan, Z.-P. Qian, Y.-S. Zhang, Y. Cai, and W.-Q. Cao, "Hybrid SIW-GCPW narrow-wall 3 dB coupler," Frequenz, vol. 67, no. 7-8, pp. 209-212, 2013.

[11] J. D. Barrera and G. H. Huff, "Analysis of a variable SIW resonator enabled by dielectric material perturbations and applications," IEEE Transactions on Microwave Theory and Techniques, vol. 61, no. 1, pp. 225-233, 2013.

[12] J. Xu, D. Zhou, D. Lv, and Y. Zhang, "A novel microwave equalizer using substrate integrated waveguide concept," in Proceedings of the China-Japan Joint Microwave Conference (CJMW'11), pp. 1-3, Hangzhou, China, April 2011.

[13] W. Huan, Millimeter wave gain equalizer [Ph.D. thesis], University of Electronic Science and Technology of China, Chengdu, China, 2013.
[14] Y. Wang, D. Zhou, Y. Zhang, and C. Chang, "Using multilayered substrate integrated waveguide to design microwave gain equalizer," Advances in Materials Science and Engineering, vol. 2014, Article ID 109247, 6 pages, 2014.

[15] Z. G. Hua and H. Wei, "Integrated wideband folded half mode substrate integrated waveguide bandpass filter," Acta Electronica Sinica, vol. 38, no. 4, pp. 825-829, 2010.

[16] Y. Wang, C. You, and X. Zhu, "Half-mode substrate integrated waveguide (HMSIW) directional filter with complementary split ring resonator (CSRR)," in Proceedings of the Asia Pacific Microwave Conference (APMC '09), pp. 2542-2544, IEEE, Singapore, December 2009.

[17] F. Chen, K. Song, B. Hu, and Y. Fan, "Compact dual-band bandpass filter using HMSIW resonator and slot perturbation," IEEE Microwave and Wireless Components Letters, vol. 24, no. 10, pp. 686-688, 2014.

[18] Y. Cassivi, L. Perregrini, P. Arcioni, M. Bressan, K. Wu, and G. Conciauro, "Dispersion characteristics of substrate integrated rectangular waveguide," IEEE Microwave and Wireless Components Letters, vol. 12, no. 9, pp. 333-335, 2002.

[19] Z. Xiong, "Transition design between substrate integrated waveguide and microstrip line," Journal of PLA University of Science and Technology, vol. 14, no. 2, pp. 129-1333, 2013.

[20] L. C. En, Basis of Microwave Technique, Xian Electronic and Science University Press, Xian, China, 2007.

[21] R. Levy, "Analysis and synthesis of waveguide multiaperture directional coupler," IEEE Transactions on Microwave Theory and Techniques, vol. 16, no. 12, pp. 995-1006, 1968. 

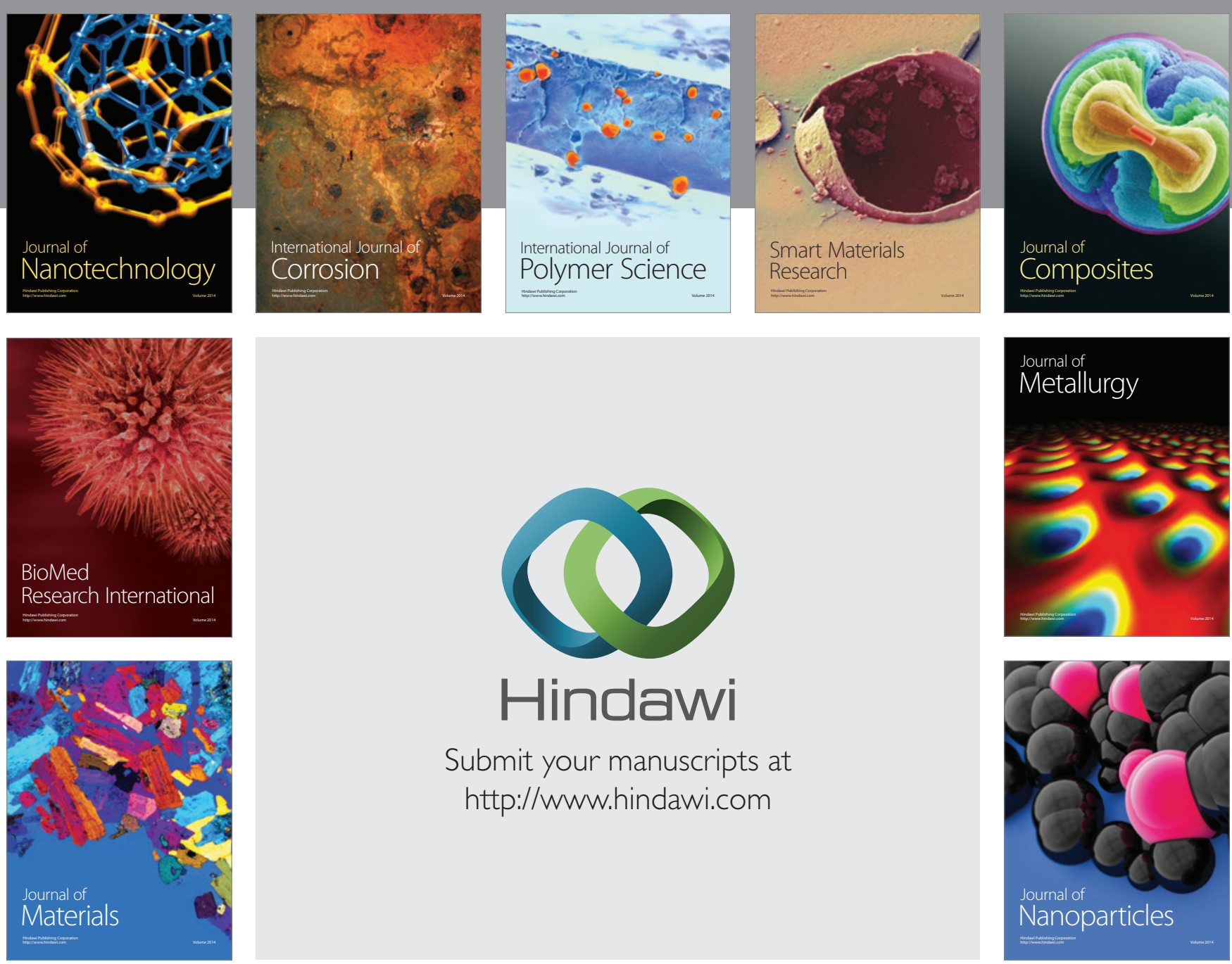

Submit your manuscripts at http://www.hindawi.com
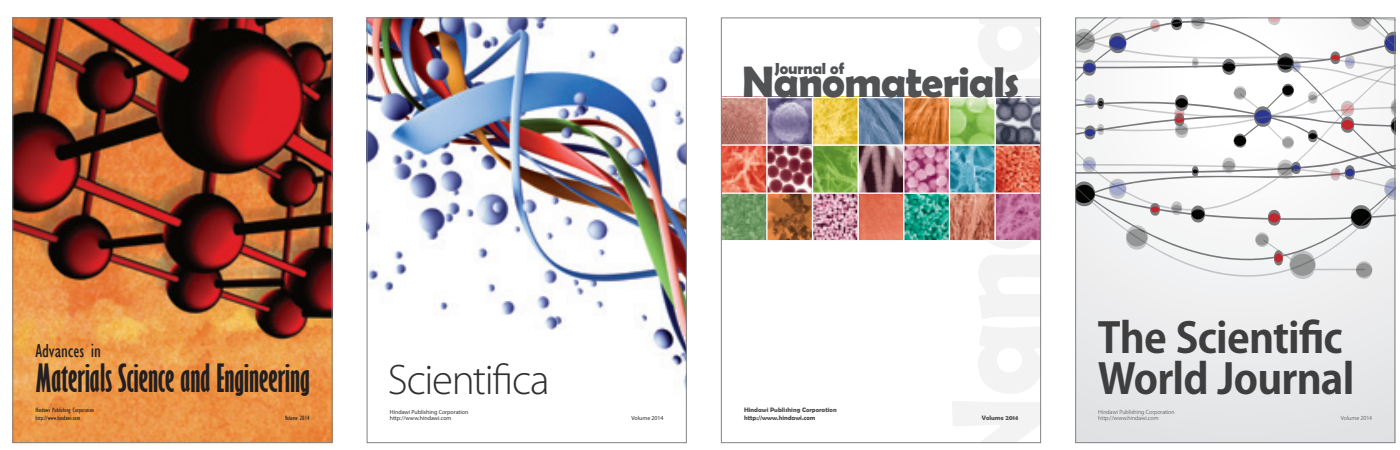

\section{The Scientific World Journal}
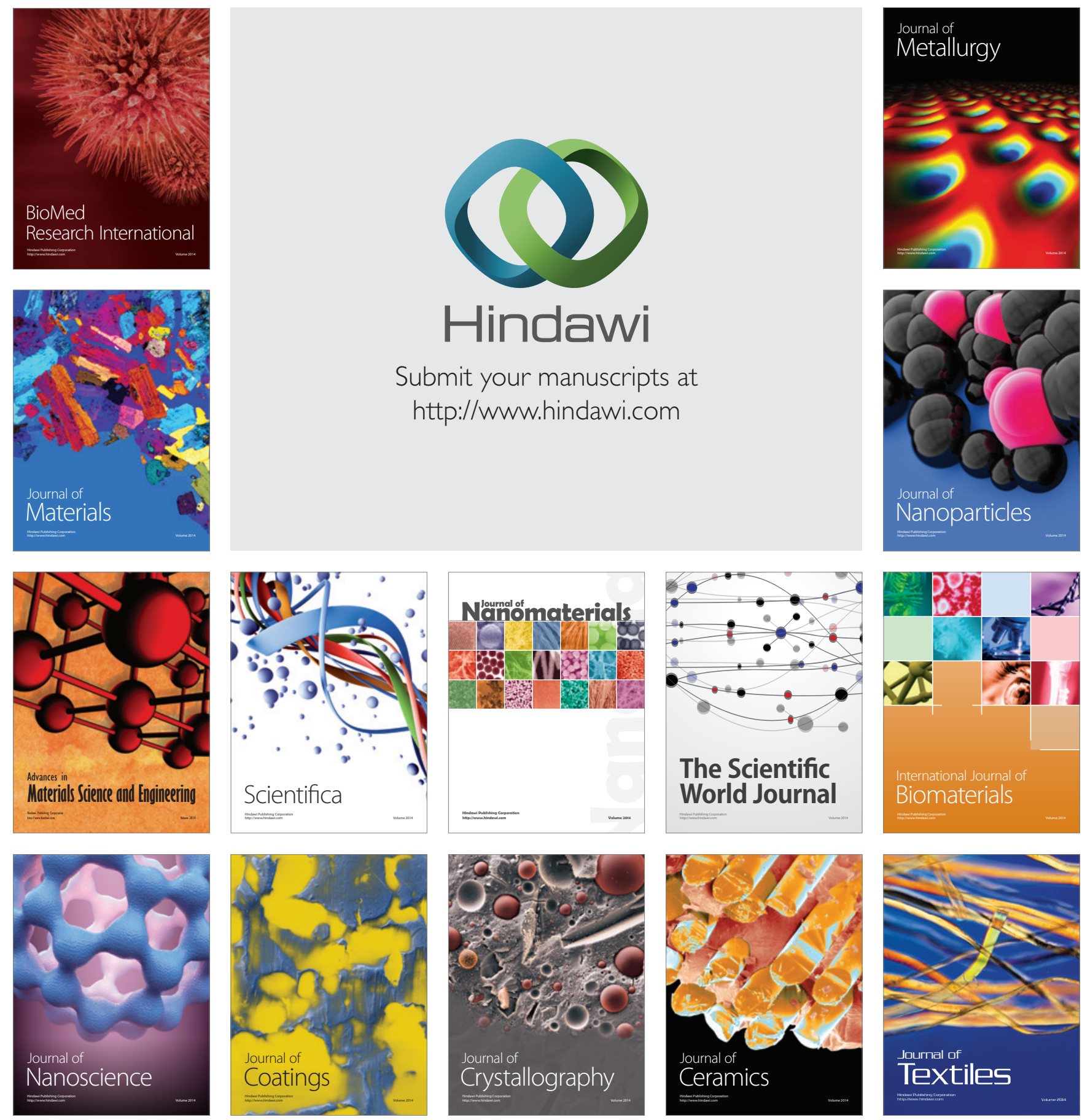